Traditional materials from new sources - conflicts in analytical methods for calcium carbonate.

Belton, David J., Hickman, Graham J. and Perry, Carole C.*

Biomolecular and Materials Interface Research Group, School of Science and

Technology, Nottingham Trent University, Clifton lane, Nottingham NG11 8NS, United Kingdom

TEL: +44 (0)115 8486695 Email: carole.perry@ntu.ac.uk 


\title{
Traditional materials from new sources - conflicts in analytical methods for calcium carbonate.
}

\begin{abstract}
Calcium carbonate, (E170) is a common food and pharmaceutical additive/ingredient. In addition to a source of calcium, the carbonate has uses including as a colour, acidity regulator and bulking agent. Globally, a range of regulatory agencies and pharmacopoeia control the analyses and specification of additives in food, supplements, pharmaceutical substances and excipients. Accordingly, a range of specifications and analyses exist for calcium carbonate depending on the application and market of the product. In this contribution, we analyse calcium carbonates from geological, synthetic and biogenic sources, focussing on acid insoluble impurities, a test required by current monographs. Analysis of calcium carbonate from different origins may require modification of existing tests to comply with regulatory bodies, due to the variation of impurities specific to the source of the material. We suggest an analytical approach involving centrifugation that improves analytical efficiency (up to $85 \%$ time reduction), especially for calcium carbonate of biological origin.
\end{abstract}

Keywords: calcium carbonate; E170; FCC; USP; pharmacopeia

\section{Introduction}

Many different legislations and global pharmacopoeia at the national and international level regulate the specification and analysis of food additives, drug substances and excipients. Calcium carbonate (E170) is used as an acidity regulator and anti - caking agent, as a food additive or as an active ingredient when added as a dietary supplement. As calcium carbonate can be classed both as an excipient and a drug substance it is regulated by global pharmacopoeias (e.g. Ph. Eur., Ph. Int. and USP) (CE 2016a; USP 2017; WHO 2017). As a permitted food additive (E170) it is regulated, for example, by the European food safety authority (EFSA), the U.S. Food and Drug Administration (FDA) and the Joint FAO/WHO Expert Committee on Food Additives (JECFA) (ANS 2011; JECFA 2006; USP 2016a). Each body sets its own limits on the purity and impurity 
profile for a given substance and specifies analytical methods for the determination of the same (table 1).

Table 1. Comparison of specifications for major calcium carbonate components and impurities between different regulatory monographs.

\begin{tabular}{l|ccccc}
\hline Test & Ph. Eur. & USP / Ph. Int. & E170 & FCC & FCC (Limestone) \\
\hline Assay (\% after drying) & $98.5-100.5$ & $98.0-100.5$ & $98.0-100.0$ & $>98.0$ & $>94.0$ \\
Acid insoluble substances (\%) & $<0.2$ & $<0.2$ & $<0.2$ & $<0.2$ & $<0.2$ \\
Magnesium and alkali salts (\%) & $<1.5$ & $<1.0$ & $<1.0$ & $<1.0$ & $<1.0$ \\
\hline
\end{tabular}

The information used to construct table one can be found in CE 2016a, WHO 2017, USP 2016a, 2016b and 2017 and Commission Regulation (EU) No 231/2012.

Many of the specified methods have been developed from a historical perspective and assume a geological source for the raw material. Thus may not be entirely fit for purpose when applied to products derived from different sources, particularly those of natural or biogenic origin. Historically, calcium carbonates do originate from geological sources (GCC). The material is mined, milled, washed and, where necessary, further purified by precipitation from calcium hydroxide to produce precipitated calcium carbonate (PCC). Carbonates from geological sources can have very different composition profiles to the biogenically derived materials $(\mathrm{BCC})$ that are increasingly entering the market as the producing industries strive to improve their 'green credentials' and reduce the cost of waste management (Chen et al. 2008; Murakami et al. 2007). Examples of biogenic sources used by the food industry include by-products of the egg processing and shellfish industries, both of which have physicochemical properties and constituents that distinguish them from mined materials, unless highly reprocessed.

The various assay limits imposed by each of the regulatory bodies for calcium carbonate 
can depend on the proposed application, for example assay limits may be as little as $94 \%$ and the content of acid insoluble substances as high as 3.5\% when calcium carbonate is labelled as a limestone derived food additive (USP 2016b). For all other applications and descriptions, the minimum assay is $98.0-98.5 \%$ of the dried material and $0.2 \%$ for the acid insoluble component (CE 2016a; Commission Regulation No 231/2012; USP 2016a; USP 2016b; USP 2017; WHO 2017). One of the major differences one may expect to find between geological and biogenic calcium carbonate is the presence of biologicals such as protein and carbohydrate, which are invariably components of the biogenic form (unless heavily purified by dissolution and precipitation). This presents major difficulties in comparing the various analytical methodologies in that they may erroneously report the presence of these components.

To highlight the differences in the methods used across the regulatory bodies and the impact on the final analytical outcome, summaries of the relevant sections from representative monographs are presented below:

- European and International pharmacopoeia monographs (CE 2016a; WHO 2017): A measured amount of test material is dissolved until effervescence ceases in an acetic acid solution, followed by boiling for $2 \mathrm{~min}$. This solution is passed through a tared sintered glass filter such as a Gooch crucible. The residue is washed with hot water and dried at $105^{\circ} \mathrm{C}$ for $1 \mathrm{~h}$. The residue should not weigh more than $0.2 \%$ of the starting material.

- Food chemicals codex (USP 2016a), United States pharmacopoeia (USP 2017) and the Joint Expert Committee for Food Additives (JECFA 2006) monographs: A measured amount of test material is dissolved until effervescence ceases in a hydrochloric acid solution, followed by boiling for 2 min, then 
digesting on a steam bath for $1 \mathrm{~h}$. After cooling this solution is passed through a filter paper. The residue is washed until no chloride is detected on the addition of silver nitrate and the filter ignited. The residue should not weigh more than $0.2 \%$ of the starting material.

As described above most regulatory bodies apply a dissolution and recovery method for determination of acid insoluble material. The European and International pharmacopoeia methods are based on filtration using sintered glass but do not specify a pore size. However, for some pharmacopoeia, the appendix cited (2.1.2) provides a comparative table of pore sizes of sintered-glass filters and indicates UK grade 4 (4 - $10 \mu \mathrm{m}$ pore diameter) as suitable for analytical work (CE 2016b). To speed up the filtration process it would be quite possible to select a coarser grade (e.g. UK grade 3, 16 - $40 \mu \mathrm{m}$ pore diameter) (CE 2016b). Depending on the chemical nature of the insoluble component, this could be quite suitable or, could equally allow some of the potentially retainable insoluble material to be lost resulting in an erroneously low insolubles determination yet this approach would still comply with the monograph methodology.

Other bodies (FCC, JECFA and USP) use an 'ashless' filter paper to retain the insoluble material, which is ignited at $550{ }^{\circ} \mathrm{C}$. The mass remaining after this treatment is used to determine the insoluble content. There are several problems with this approach. The protocols (FCC, JECFA and USP) fail to specify a filter grade other than 'ashless' and as pore diameters of such filters can range from $2.5 \mu \mathrm{m}$ to $25 \mu \mathrm{m}$ a wide range of retained material is possible. Manufacturer specifications give some insight into the suitability of the various grades and accordingly we selected Whatman no. 42 'ashless' having the smallest pore diameter $(2.5 \mu \mathrm{m})$ of the 'ashless' filter papers for our gravimetric analyses. A further issue with the use of thermal treatment as high as $550{ }^{\circ} \mathrm{C}$ in the ignition method increases the likelihood of decomposition of the retained acid insoluble material 
particularly where it is biological in nature. Thus, calcium carbonate originating from sources higher in such impurities has the potential to give very different results by the different applied methods.

The first serious difficulty encountered when comparing calcium carbonate from different sources is in filtering the biogenic residues as these tend to block the pores in the filter types used ('ashless' paper and glass sinter are equally compromised) and in our experience this can result in delays of up to $48 \mathrm{~h}$ in the analytical process. Secondly, and more importantly, the ignition method will completely volatilise residual bio-organics such as proteins and carbohydrates. If present in quantities greater than $1.5-2.0 \%$ of the total mass, they will show up as a fail in the calcium carbonate assay method $(<98.0-$ 98.5\%). If present at levels lower than this there is a potential for the material to pass erroneously both the assay and the acid insoluble tests with the remaining required tests not able to pick up this discrepancy (see table 1).

For the reasons outlined above, we have developed a less ambiguous method which allows us to quantify the acid insoluble fraction of calcium carbonate more efficiently and which does not disguise the presence of organic matter by combustion.

To demonstrate this approach, samples of calcium carbonate of various origins were analysed by the Ph. Eur. (drying) method, the JEFCA (ignition) method and the results compared after applying our approach; a centrifugation method to the same samples. All results were treated statistically in the same way ( 5 replicates of all samples and applying a single standard deviation to compare the reproducibility of the data). 


\section{Methodology}

\subsection{Materials:}

Hydrochloric acid 37\% and Acetic acid glacial, Fisher Scientific (Loughborough, UK). Silver Nitrate $>99 \%$ and calcium carbonate standard (cat. \# 12010), Sigma Aldrich (Gillingham, UK). Ethanol absolute 100\% Haymankimia (Witham, UK). Ashless filter papers, Whatman no 42, GE Healthcare, (Buckinghamshire, UK). A range of food-grade calcium carbonate samples intended for the UK supplements market were obtained and tested as received, including agglomerated calcium carbonates (C001 and C002), precipitated calcium carbonates (C003 and $\mathrm{C} 004)$ and a biogenic calcium carbonate (C005).

\subsection{Methods to determine acid insoluble substances:}

2.2.1 Recovery of acid insolubles by glass sinter filtration (Ph. Eur.):

A $5.00 \pm 0.01 \mathrm{~g}$ sample was dissolved in $80 \mathrm{~mL}$ of $12 \%$ acetic acid. When effervescence ceased, the sample was boiled for $2 \mathrm{~min}$. After cooling, the solution was diluted to 100 $\mathrm{mL}$ with $12 \%$ acetic acid and filtered through a sintered-glass filter (grade 4 ). The residue obtained was washed 4 times with $5 \mathrm{~mL}$ of hot water and dried at $100-105^{\circ} \mathrm{C}$ for $1 \mathrm{~h}$. The residue was weighed and compared with the limit of $0.2 \%$ (10 $\mathrm{mg}$ for $5 \mathrm{~g}$ of sample).

\subsubsection{Recovery of acid insolubles by ashless paper (USP - JECFA):}

A $5.00 \pm 0.01 \mathrm{~g}$ sample was suspended in $25 \mathrm{~mL}$ of water to which was added $25 \mathrm{~mL}$ of a 1 in 2 solution of hydrochloric acid (37\%) and made up to $200 \mathrm{~mL}$ with $18 \mathrm{M} \Omega ~ \mathrm{H}_{2} \mathrm{O}$. When effervescence ceased, the solution was digested on a steam bath for $1 \mathrm{~h}$, allowed to cool and filtered using quantitative ashless paper (grade no. 42 Whatman). The precipitate was washed with $18 \mathrm{M} \Omega \mathrm{H}_{2} \mathrm{O}$ until no chloride was detected with a silver nitrate (4.2 
$\mathrm{w} / \mathrm{v}$ ) solution and the paper ignited in an ashing furnace at $550{ }^{\circ} \mathrm{C}$ to constant weight. The residue was weighed and compared with the limit of $0.2 \%$.

\subsubsection{Acid insolubles by centrifugation:}

A $5.00 \pm 0.01 \mathrm{~g}$ sample was dissolved in $80 \mathrm{~mL}$ of $12 \%$ acetic acid. When effervescence ceased, the sample was boiled for 2 min. After cooling, the solution was diluted to 100 $\mathrm{mL}$ with $12 \%$ acetic acid and transferred to 2 tared $50 \mathrm{~cm}^{3}$ polypropylene Falcon centrifuge tubes and the insolubles sedimented by centrifugation at $2880 \mathrm{rcf}$ for $10 \mathrm{~min}$ using an Eppendorf 5804R centrifuge equipped with a A-4-44 swing bucket rotor. The supernatant was removed, and the sediment was washed with ethanol in deionised water (1:2) and sedimented as before. The addition of ethanol was to promote sedimentation of the supernatant and eliminate adhesion of particulates to the plastic walls of the centrifuge tubes. The wash process was repeated a further 2 times and then the tubes and residues were dried at $80^{\circ} \mathrm{C}$ to constant weight.

\subsection{Data analysis:}

All calculations were performed using Excel 2013 (Microsoft Office Windows). All analyses were carried out on five replicates and the results presented as means \pm standard deviation (SD).

\subsection{Characterisation of insoluble materials generated}

Residues were analysed by both spectroscopic and thermogravimetric approaches to identify the composition of the insoluble fraction.

2.4.1 Fourier transform infrared attenuated total reflectance spectroscopy (FTIR-ATR):

Residues from each treatment were analysed using an Agilent Cary 630 equipped with a 
diamond single bounce ATR accessory. Accumulation of 16 individual spectra from 4000 - $650 \mathrm{~cm}^{-1}$ at $4 \mathrm{~cm}^{-1}$ resolution was performed with the instrument background automatically subtracted.

\subsubsection{Thermogravimetric analysis (TGA):}

Samples were analysed using a Mettler Toledo TGA/SDTA851e instrument under a constant supply of clean, dry compressed air. The program used was to hold at $30{ }^{\circ} \mathrm{C}(10$ min) then ramp from $30-550{ }^{\circ} \mathrm{C}$ at $10{ }^{\circ} \mathrm{C}$ per min before holding at $550{ }^{\circ} \mathrm{C}$ for $2 \mathrm{~h}$. Data analysis was performed using STARe software V15 build 8668.

\section{Results and Discussion}

Samples were obtained of precipitated calcium carbonates (C001 to 4) and a calcium carbonate of biogenic origin (C005). In addition, a sample of calcium carbonate compliant with the Ph. Eur., BP, USP and FCC, E170 monographs was purchased from Sigma Aldrich (cat.\# 12010 batch\# SZBE3280V), this was designated Sigma.

Several issues were observed during application of the established acid insoluble analytical methods used. Though most of the stages (dissolution, digest, drying or ignition) were similar and predictable for all methods and samples, processing times differed (table 2) at the insoluble isolation stage. The filtration methods were generally quicker using sintered glass (grade 4, 10-16 $\mu \mathrm{m}$ pores) than 'ashless' filter paper (Whatman no. $42,2.5 \mu \mathrm{m}$ pores) due both to the different pore diameters and the application of reduced pressure with the sinters. In addition, the washing time required to remove residual chloride was considerably extended when using the filter papers. Both filtration methods were extremely slow when C005 was processed, with up to 3 days being required to complete the filtration and washing stages due to the large amount of 
insoluble material generated quickly blocking the filter pores. This issue could be improved by intermittent ultra-sonication of the sintered glass filters without affecting the results (supplementary data table $\mathrm{S} 1$ ) but the time required was still felt to be excessive $(>8 \mathrm{~h})$.

Improvements at sample recovery stage were the main motivation for the method modification. Rather than using filters to isolate the insoluble components we decided to use sedimentation accelerated by centrifugation. All the samples sedimented within 10 min at $2880 \mathrm{rcf}$ in $50 \mathrm{~mL}$ plastic (polypropylene) 'Falcon' centrifuge tubes and the addition of ethanol (30\%) to the wash solvent facilitated both sedimentation and prevented adhesion of the insolubles to the plastic walls of the tubes. Due to the chemical nature of the centrifuge tubes used we selected a drying temperature of $80^{\circ} \mathrm{C}$, a temperature at which the tubes were shown to be stable gravimetrically (supplementary data fig. S1). Under this regime the acid insoluble fraction of all sample types could be isolated within half a working day, a time saving of $\sim 85 \%$ in the case of the biogenically sourced sample C005.

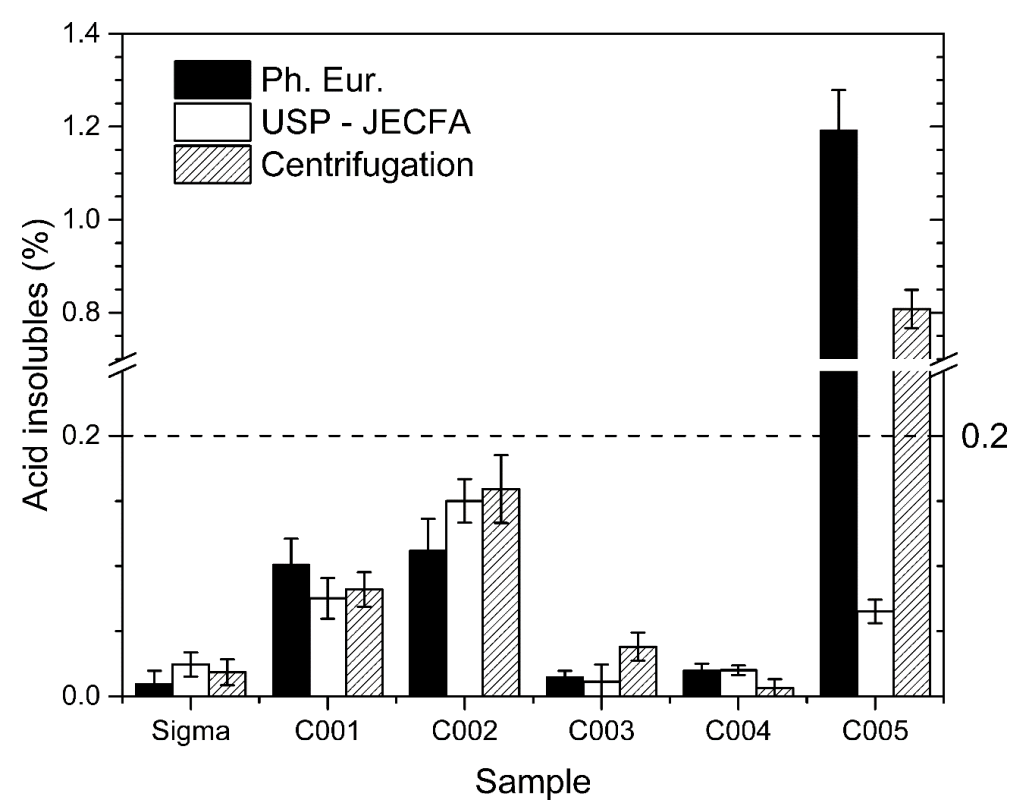


Figure 1. Acid insolubles by gravimetric analysis by $\mathrm{Ph}$. Eur., USP/JECFA and centrifugation. Error bars show $1 \sigma$ standard deviation from the mean result of 5 replicates. The line at $0.2 \%$ represents the limit for acid insolubles compliance with the different monographs.

Table 2. Results for each acid insoluble test for each material tested, represented as the average of five replicate tests \pm standard deviation.

\begin{tabular}{lcccccc}
\hline Sample & \multicolumn{3}{c}{ Acid insoluble content (\%) } & \multicolumn{3}{c}{ Total analysis time (days) } \\
\hline & Ph. Eur. & USP & Centrifuge & Ph. Eur. & USP & Centrifuge \\
\hline Sigma & $0.01 \pm 0.01$ & $0.02 \pm 0.009$ & $0.02 \pm 0.01$ & 1.0 & 1.0 & 0.5 \\
C001 & $0.10 \pm 0.019$ & $0.08 \pm 0.016$ & $0.08 \pm 0.013$ & 1.0 & 1.0 & 0.5 \\
C002 & $0.11 \pm 0.024$ & $0.15 \pm 0.017$ & $0.16 \pm 0.026$ & 1.0 & 1.0 & 0.5 \\
C003 & $0.01 \pm 0.005$ & $0.01 \pm 0.013$ & $0.04 \pm 0.011$ & 1.0 & 1.0 & 0.5 \\
C004 & $0.02 \pm 0.005$ & $0.02 \pm 0.004$ & $0.01 \pm 0.007$ & 1.0 & 1.0 & 0.5 \\
C005 & $* 1.19 \pm 0.086$ & $0.07 \pm 0.009$ & $* 0.81 \pm 0.041$ & 3.0 & 2.0 & 0.5 \\
\hline
\end{tabular}

Values preceded by an * would not be considered as passes under the testing criteria. Also presented is the total time (days) required to complete a test for each of the methods applied for each of the samples tested.

A comparison of the analytical results obtained (fig. 1 and table 2) show that we found good correlation between the $\mathrm{Ph}$. Eur. and our centrifugation method, both of which use relatively mild thermal treatments for drying. The most notable observation being the failure (by a large margin - see fig. 1 and table 2) of compliance for the biogenically sourced material (C005) when tested without ignition (Ph. Eur. and centrifugation methods), whilst the USP ignition method passed this material comfortably. The reason for the ignition step in the USP method is to ensure complete combustion of the ashless filter paper and ideally recover the acid insoluble residues unaltered, a situation that would be expected in samples derived from mineral and chemical sources, but which does 
not hold necessarily for samples of a biogenic origin.

Further, to determine the chemical nature of the residues isolated by the dissolution processes infrared analysis (FTIR-ATR) was conducted on all the residues isolated by the centrifugation method (chosen initially as the least thermally damaging process) and compared to samples generated by ignition where significant gravimetric differences were observed. Due to interference from the sinter during recovery, FTIR analysis of the residue for the Ph. Eur. method was not possible (fig. S4). The reference material (Sigma) did not provide any isolable material useful for this purpose so no data was generated. Notably the isolates give a spectroscopic signature indicative (though not conclusive) of their source. C001 and C002 both gave the signature of dolomite (Calcium magnesium carbonate $\mathrm{CaMg}\left(\mathrm{CO}_{3}\right)_{2}$ (fig. 2a)), which has a significant frequency shift of the in-plane $\mathrm{C}-\mathrm{O}$ bending vibration peak which appears at $712 \mathrm{~cm}^{-1}$ in calcite and $727 \mathrm{~cm}^{-1}$ in dolomite (supplementary data fig. S2) indicative of a mineral origin (Correia et al. 2015; Lafuente et al. 2015). Although a carbonate, dolomite does not dissolve as rapidly in dilute hydrochloric and acetic acids as calcite and clearly, it appears to have survived the dissolution process employed here. However, at the levels found it is not expected to interfere significantly with the assay to determine sample purity nor the magnesium and alkali salt determinations. Due to the limited isolable material from C003 and C004 the spectral quality was somewhat reduced but there is sufficient evidence to suggest most of the acid insoluble material from these to be a silicate mineral, again the levels recorded do not suggest any interference in any of the other tests required for compliance. 

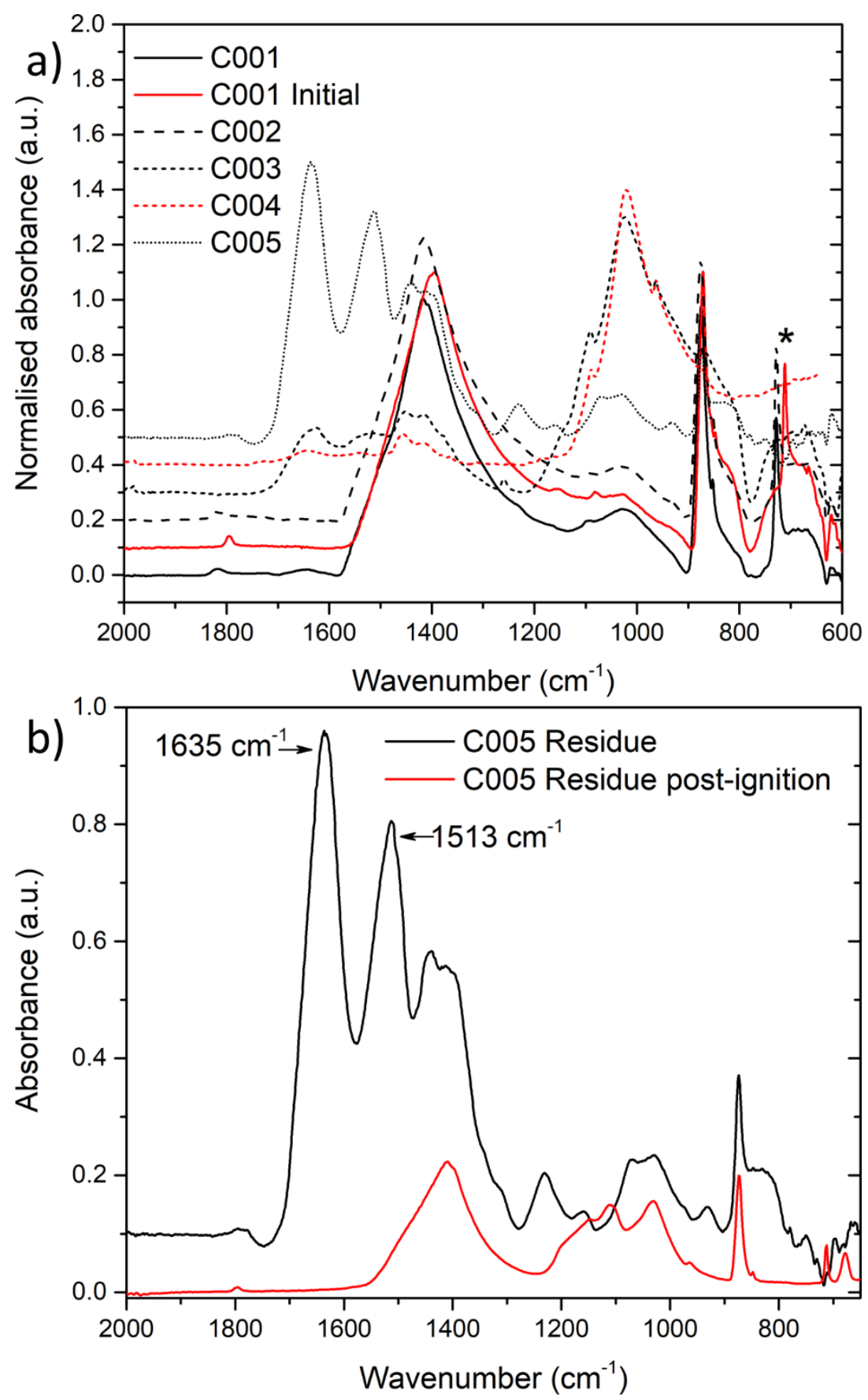

Figure 2. Acid insolubles isolated by centrifugation, (a) infrared spectra of residues C001, untreated C001, C002, C003, C004 and C005. The location of the peak shift for dolomite is indicated by an asterisk. (b) The infrared spectra of residues C005 before and after ignition.

The spectrum of C005 (fig. 2b) is typical of a protein/peptide (the 1630 and $1515 \mathrm{~cm}^{-1}$ bands are characteristic of amide I and amide II for polypeptides), suggesting a sample 
biogenic in origin (Barth 2007). A spectrum collected of the same sample post ignition showed the complete removal of the protein component and that this acid insoluble fraction contained residual calcite presumably having been protected from dissolution by the presence of the protein. Thermogravimetric analysis of this sample (fig. 3) to mimic the ignition process showed the majority of the material to be combustible under the conditions of the USP method. The TGA data also suggests a shared nature and potentially origin of $\mathrm{C} 001$ and $\mathrm{C} 002$ and $\mathrm{C} 003$ and $\mathrm{C} 004$ with the isotherms showing close resemblance to each other, corroborative evidence for the trends in the spectra of the residues observed by infrared analysis. When continued to constant weight (supplementary data fig. S3) the final residual component of this fraction reduced by $\sim 94 \%$, which correlates well with the observed discrepancy between the ignition method (USP/JECFA), and the drying methods (Ph. Eur. and centrifugation). This level of error would be expected to have a significant implication for assay determination in the case of this sample but if up to $1.5 \%$ of the sample were acid insoluble but combustible, the material could still pass all the tests when applying the USP/JECFA monograph methods. 

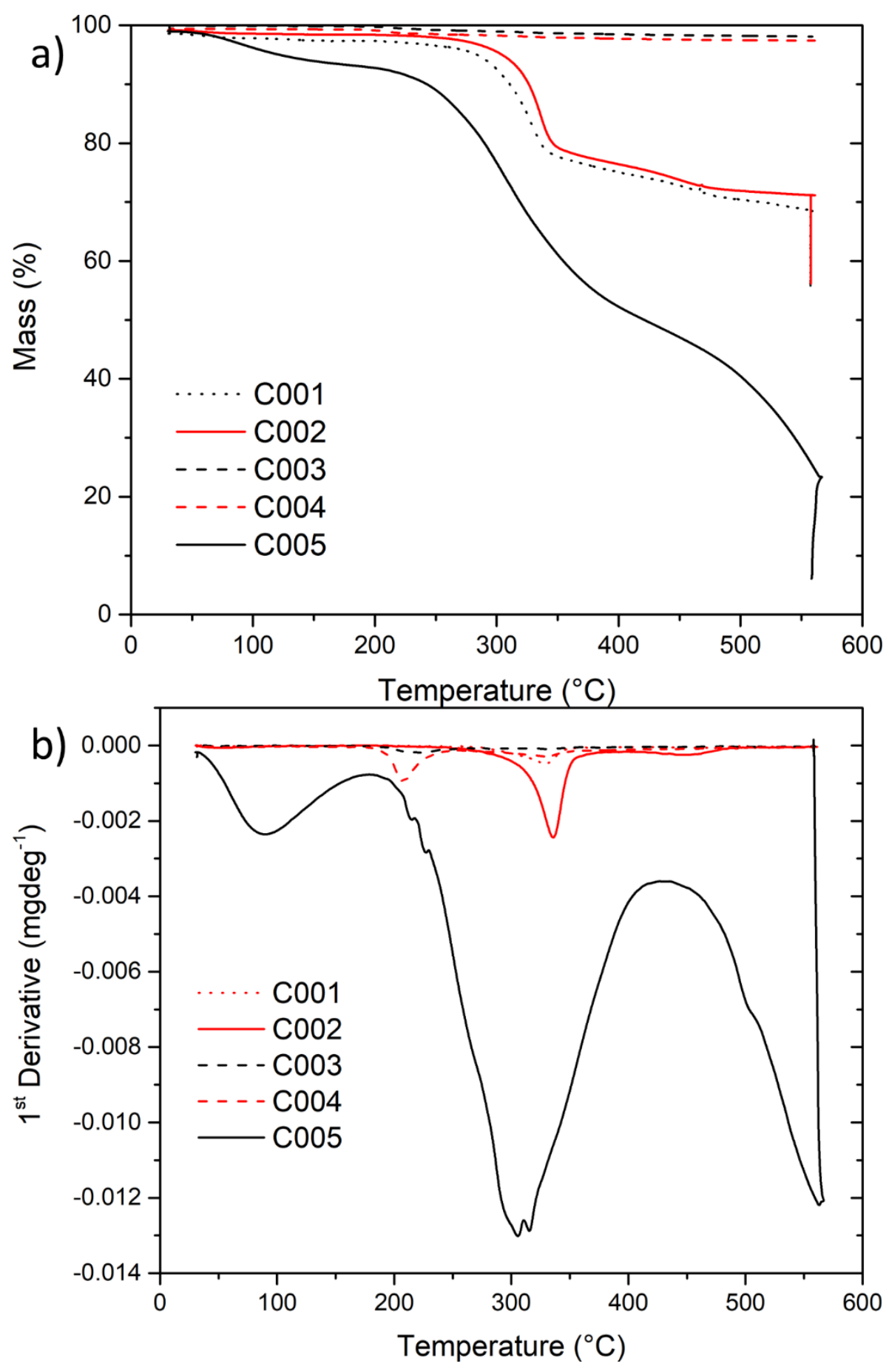

Figure 3. Thermogravimetric analysis of the residues from the $\mathrm{Ph}$. Eur. process a) percentage remaining b) 1st derivative.

Where calcium carbonate purity is defined by ignition methods, such as in European regulation for E170, as well as USP and JECFA monographs our results suggest there is a risk that the level of acid insoluble impurities for biogenic materials may be significantly 
greater than that defined in the monograph. Depending on the nature of the impurities, the higher than permitted level of acid insoluble material may not be detected by a supporting assay, such as complexometric titration (CE 2016a; Commission Regulation No 231/2012; USP 2016a; USP 2017; WHO 2017). Most monographs set a limit for acid insolubles of $0.2 \%$ (table 1), the limit of assay purity is generally $98.0 \%$, and as such acid insoluble impurities could be up to a factor of $10 \mathrm{x}$ higher when relying on ignition methods yet still comply with the monograph limits.

The implications of this study is dependent on the source of the material under test and the specific composition of the impurities. For the eggshell derived material tested in this study the organic proteinaceous impurities identified in biogenic carbonate are probably benign with hydrolysed eggshell membrane being used as a supplement for healthcare applications (Ruff et al. 2009). However, with organic impurities of biological origin there is a risk of increased exposure to potentially allergenic material for the consumer, especially when the source of the material is egg as it is a notifiable allergen in geographic regions such as the EU. We note, though that for analysis of food grade material there is reliance on ignition methods to identify impurities of this type (Allen et al. 2014).

Further, when we consider the wide range of polymeric materials including plastics such as polyethylene, polyethylene terephthalate and nylon which undergo at least partial decomposition at temperatures up to $500{ }^{\circ} \mathrm{C}$, (Ray and Cooney 2012) we must acknowledge that there is a large number of potential acid insoluble impurities that are challenging to detect by the ignition method used today in geographic regions using Commission Regulation No 231/2012, USP and JECFA methods.

\section{Conclusions}

For two common methodologies for the detection of acid insoluble substances in food 
and pharmaceutical grade calcium carbonate (E170), a serious discrepancy was found when applying these methods to calcium carbonate of biogenic origin. We confirmed the reason for the apparent compliance of this non-compliant material when examined according to the USP and JECFA monograph procedures and identified the acid insoluble components spectroscopically providing further evidence as to the materials origins. Universal adoption of the Ph. Eur. methodology would resolve the discrepancy when analysing calcium carbonates with high organic content. However, the adoption of the centrifugal method presented as a new analytical methodology for screening acid insoluble content would represent a significant improvement in methodological clarity and a saving of over $48 \mathrm{~h}$ in processing time for the analysis of samples containing large quantities of acid insoluble material. The new centrifugation-based recovery method described here provides results of a similar accuracy and reproducibility as that of the $\mathrm{Ph}$. Eur. method for calcium carbonate for all the origins trialled whether compliant or noncompliant. As biogenic carbonates become more popular due to their renewable nature and as waste valorisation plays a larger role in the modern economy, the analytical methods for analysis of materials from novel sources will need to be changed or amended.

\section{Acknowledgements}

The authors would like to acknowledge funding provided by Innovate UK (EPSRC EP/N0208950/1) and the UK Technology Strategy Board - Nutrition for Life programme.

\section{Conflict of interest}

The authors declare no conflict of interest.

\section{References}

Allen KJ, Turner PJ, Pawankar R, Taylor S, Sicherer S, Lack G, Rosario N, Ebisawa M, Wong G, Mills C, et al. 2014. Precautionary labelling of foods for allergen 
content: are we ready for a global framework? World Allergy Organ. J. 7(1):1-10. doi.org/10.1186/1939-4551-7-10

Barth A. 2007. Infrared spectroscopy of proteins. Biochim. Biophys. Acta. 1767: 10731101. doi:10.1016/j.bbabio.2007.06.004.

Chen HS, Chang JH, Wu JSB. 2008. Calcium bioavailability of nanonized pearl powder for adults. J. Food Sci. 73: H246-H251. doi:10.1111/j.1750-3841.2008.00965.x

Correia LM, de Sousa Campelo N, Novaes DS, Cavalcante CL, Cecilia JA, RodríguezCastellón E, Vieira RS. 2015. Characterization and application of dolomite as catalytic precursor for canola and sunflower oils for biodiesel production. Chem. Eng. J. 269: 35-43. doi:10.1016/j.cej.2015.01.097

[CE] Council of Europe. 2016a. Calcium carbonate. In Council of Europe, European Pharmacopoeia. ( $9^{\text {th }}$ edition). Strasbourg: Council of Europe, p. 1912.

[CE] Council of Europe. 2016b. Comparative table of glass sinters (2.1.2). In Council of Europe, European Pharmacopoeia. ( $9^{\text {th }}$ edition). Strasbourg: Council of Europe, (p. 15).

Commission Regulation (EU) No 231/2012 of 9 March 2012 laying down specifications for food additives listed in Annexes II and III to Regulation (EC) No 1333/2008 of the European Parliament and of the Council.

[ANS] EFSA Panel on Food Additives and Nutrient Sources added to Food. 2011. Scientific opinion on re-evaluation of calcium carbonate (E 170) as a food additive. EFSA J. 9: 2318-2391. doi:10.2903/j.efsa.2011.2318.

[JECFA] Joint FAO/WHO Expert Committee on Food Additives. 2006. Calcium carbonate. In Joint FAO/WHO Expert Committee on Food Additives. Combined compendium of food additive specifications. Rome: World Health Organization: Food and Agriculture Organization of the United Nations.

Lafuente B, Downs RT, Yang H, Stone N. 2015. The power of databases: the RRUFF project. In: Armbruster T, Danisi RM editors. Highlights in mineralogical crystallography. Berlin: W. De Gruyter, p. 1-30.

Murakami FS, Rodrigues PO, Campos CMT, Silva MAS. 2007. Physicochemical study of $\mathrm{CaCO}_{3}$ from egg shells. Food Sci. Technol. 27: 658-662. doi:10.1590/S010120612007000300035

Ray S, Cooney RP. 2012. Thermal degradation of polymer and polymer composites. In Kutz M. editor. Handbook of environmental degradation of materials ( $2^{\text {nd }}$ edition). Oxford: William Andrew Books, p. 213-242. 
Ruff KJ, DeVore DP, Leu MD, Robinson MA. 2009. Eggshell membrane: A possible new natural therapeutic for joint and connective tissue disorders. Results from two open-label human clinical studies. Clin. Interv. Aging. 4: 235-240.

[USP] The United States Pharmacopeial Convention Inc. 2016a. Calcium carbonate. In United States Pharmacopeial Convention Inc., Food Chemicals Codex (FCC). (10 ${ }^{\text {th }}$ edition). Rockville: The United States Pharmacopeial Convention Inc. p. 213-214.

[USP] The United States Pharmacopeial Convention Inc. 2016b. Limestone, Ground. In United States Pharmacopeial Convention Inc., Food Chemicals Codex (FCC). (10 ${ }^{\text {th }}$ edition). Rockville: The United States Pharmacopeial Convention Inc. p. 754.

[USP] The United States Pharmacopeial Convention Inc. 2017. Calcium carbonate. In United States Pharmacopeial Convention Inc., USP 41 NF 36. Rockville: The United States Pharmacopeial Convention Inc. p. 630-632.

[WHO] World Health Organization. 2017. Calcium carbonate. In World Health Organization, The international pharmacopoeia ( $7^{\text {th }}$ edition). Geneva: World Health Organization 